\title{
ORDINATION ET CLASSIFICATION DE LA VEGETATION DES ZONES HUMIDES DU SUD-EST DE LA CÔTE D'IVOIRE
}

\author{
M. L. O. KOUAME ${ }^{1}$, M. W. EGNANKOU ${ }^{1}$ et D. TRAORE ${ }^{1}$ \\ ${ }^{1}$ Université de Cocody, Laboratoire de Botanique, UFR Biosciences, 06 BP 2818 Abidjan 06. \\ E-mail : marie_ode@hotmail.com
}

\begin{abstract}
RESUME
Cette étude a porté sur l'analyse des relevés phytosociologiques effectués dans 28 stations en zones humides au Sud-Est de la Côte d'Ivoire. La matrice de données utilisée pour les analyses numériques a été constituée de 252 relevés et 398 espèces. L'analyse en composantes principales, la classification ascendante hiérarchique et l'analyse des espèces indicatrices ont été utilisées pour générer la classification des communautés végétales des zones humides. Le type de végétation dominante (herbacée ou boisée), l'hydromorphie, la salinité et le type de sol ont été les gradients environnementaux ayant permis de discriminer les communautés végétales étudiées. Ainsi, 22 communautés végétales ont été discriminées. Les espèces indicatrices des zones humides boisées du Sud-Est de la Côte d'Ivoire appartiennent à la classe des Mitragynetea Schmitz 1963, tandis que celles des zones humides herbacées, appartiennent aux classes des Phragmitetea Tüxen \& Pressing 1942 et des Potametea Tuxen et Preising 1942.
\end{abstract}

Mots-clés : Espèces indicatrices, ordination, classification, zones humides, Côte d'Ivoire.

\section{ABSTRACT}

ORDINATION AND CLASSIFICATION OF WETLAND VEGETATION IN SOUTH-EASTERN CÔTED'IVOIRE

This paper analyses vegetation data from 28 sites of wetland in South-eastern part of Côte d'lvoire. The datasets used for the analysis comprised 252 plots and 398 species. Principal Component Analysis, Cluster Analysis and Indicator Species Analysis were used to generate the wetland plant communities' classification. The dominant plants type, water level, salinity and soil types were the major environmental factors that best discriminate wetland plant communities. The different analysis of the data yielded 22 plant communities. Indicators species of the woody wetland in southeast Côte d'lvoire belongs to the Mitragynetea Schmitz 1963 while herbaceous wetland indicator species belong to the Phragmitetea Tüxen \& Pressing 1942 and the Potametea Tuxen \& Preising 1942.

Keys words : Indicator species, ordination, classification, wetland, Côte d'Ivoire. 


\section{INTRODUCTION}

La définition des communautés de plantes est un sujet qui intéresse beaucoup les scientifiques. Plusieurs techniques et approches de classification des communautés végétales ont été ainsi développées. Ces systèmes de classification ont fait l'objet de revues par divers auteurs (Shimwell, 1971; Whittaker et Gauch, 1973 ; Mueller-Dombois et Ellenberg, 1974 ; Ecological Society of America, 2004).

Les méthodes d'analyse de gradients directe ou indirecte permettent de connaître les types de végétation avec leur composition floristique en fonction des gradients environnementaux ou géographiques (Gauch, 1982 ; Kent et Coker, 1992 ; McCune et al., 2002 ; Podani, 2000).

Dans les zones humides, l'étude des communautés végétales et des gradients environnementaux s'avère très importante en raison de leur position intermédiaire entre les écosystèmes aquatiques et terrestres ainsi que des contextes géographiques et géomorphologiques où elles peuvent être rencontrées. Les méthodes d'analyses multivariées pour l'ordination et la classification de la végétation ont déjà été utilisées avec succès pour la recherche de gradients écologiques dans la végétation des zones humides (Hoagland, 2002).

En Afrique, ces méthodes d'analyse ont été utilisées pour l'étude des forêts tropicales humides (Sokpon, 1995 ; Kokou, 1998) et des savanes (Sinsin, 1993 ; Senterre, 2005). En ce qui concerne la végétation des forêts ripicoles, des études ont été réalisées suivant cette approche, notamment au Bénin, par Sokpon et al. (2001), Natta (2002) et Adomou (2005).

En Côte d'Ivoire, les études sur la végétation des zones humides ont été effectuées par les méthodes classiques. La méthode des transects est la plus couramment employée (Egnankou, 1985, Traoré, 1985 ; Houenon, 1989 ; KonanBrou et al., 1992 ; Kouamé, 1999 ; Sankaré et al., 1999). Les savanes marécageuses ont été étudiées suivant une approche physionomique par Adjanohoun (1962). Guillaumet (1967) a aussi mis en évidence les groupes aquatiques et sub-aquatiques dans la région du Bas-Cavally suivant la méthode phytosociologique de BraunBlanquet.

Cet article a pour but de montrer si l'association des méthodes d'analyse multivariées aux méthodes phytosociologiques classiques permettent de discriminer les formations végétales et des communautés végétales des zones humides du Sud-Est de la Côte d'Ivoire, ainsi que des espèces caractéristiques et des gradients écologiques principaux qui les régissent.

\section{MATERIEL ET METHODES}

\section{ZONE D'ETUDE}

La zone étudiée fait partie de la région du SudComoé qui est l'une des 19 régions administratives de la Côte d'Ivoire située au Sud-Est du pays (Figure 1 ).

Le réseau hydrographique comporte en plus de l'océan atlantique au Sud, un important système lagunaire comprenant les lagunes Ebrié, Aby et de petites lagunes comme la lagune Hébé et Kodjoboué. Les principaux cours d'eau sont le fleuve Comoé, les rivières Bia et la Tanoé.

Le relief est caractérisé par des hauts plateaux (30 m d'altitude environ) au Nord des lagunes, des bas-plateaux dont l'altitude maximale se situe entre 10 et $12 \mathrm{~m}$ et des cordons littoraux aux côtes sableuses au Sud (SECABRL/BDPA/RCT, 2004).

Le climat de la région est du type sub-équatorial, caractérisé par l'abondance des précipitations (supérieures à $2000 \mathrm{~mm}$ par an), par des températures élevées constantes $\left(26,5^{\circ} \mathrm{C}\right.$ en moyenne à Adiaké) et par des amplitudes thermiques faibles (1,6 maximum à Adiaké). La région du Sud-Comoé est située dans le domaine phytogéographique Guinéen. La végétation est diversifiée et comprend des savanes littorales, des mangroves, des forêts marécageuses, des forêts ripicoles, des forêts sempervirentes du type Diospyros sp. et Mapania spp., Thuraeanthus africanus et Heisteria parvifolia et la forêt à Eremospatha macrocarpa et Diospyros mannii. 


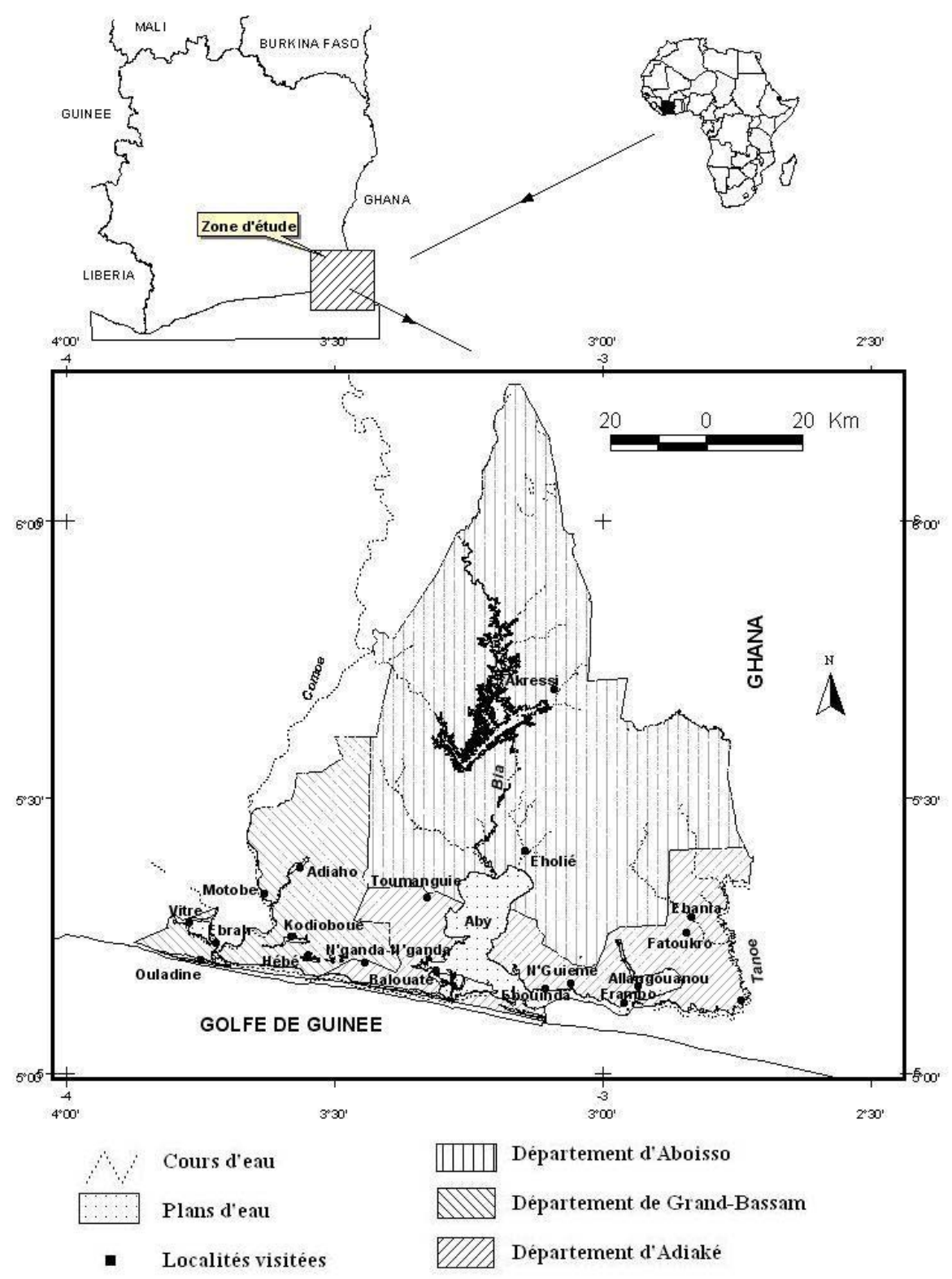

Figure 1 : Carte de la zone d'étude et de quelques localités visitées

Study area map with location of some sites visited

\section{METHODES D'ETUDE}

\section{Inventaires phytosociologiques}

Les inventaires phytosociologiques ont été effectués sur 28 sites de zones humides de la région.

A chaque site, ont été choisies des stations homogènes et représentatives où les relevés ont été effectués suivant la méthode classique de Braun-Blanquet. Les coordonnées géographiques de chaque site ont été prises par un GPS (Global Positioning System). Compte tenu de la diversité des habitats pris en compte dans l'étude, les relevés ont été stratifiés comme le suggère Van der Maarel et al. (1987). Le type de végétation selon la classification de Yangambi (Aubréville, 1957), a été assigné à chacun des relevés.

Dans les quadrats, les espèces présentes ont été notées avec leur coefficient d'abondancedominance selon l'échelle classique de Braun Blanquet.

En bordure des cours d'eau, dans les stations de forêts ripicoles, les observations ont été effectuées à l'intérieur de parcelles rectan- 
gulaires de $500 \mathrm{~m}^{2}$ comme celles utilisées au Bénin par Natta (2003). Les relevés sont équidistants de $100 \mathrm{~m}$.

Dans les mangroves, les échantillons ont été pris sur des surfaces contigües de $10 \mathrm{~m} \times 10 \mathrm{~m}$ établies le long de transects de longueurs variables, allant de l'eau libre aux endroits à hydromorphie temporaire.

Les relevés des forêts marécageuses ont été effectués sur des surfaces de $20 \mathrm{~m} \times 20 \mathrm{~m}$, sur des lignes de transects. Les relevés sont équidistants de $10 \mathrm{~m}$.

Pour les formations herbacées (prairies, végétation flottante, savanes herbacées), les échantillons ont été pris sur des surfaces de relevés de $5 \mathrm{~m} \times 5 \mathrm{~m}$ le long de transects de longueurs variables.

Des informations générales sur la texture et le $\mathrm{pH}$ des sols, la topographie (plateau, pente, vallée), le type d'hydromorphie, la salinité de l'eau, ont été notées sur les relevés.

Les observations ont été effectuées sur environ 4 ans (de Janvier 2002 à Septembre 2005) à différentes période de l'année : saison sèche (Février ou Septembre), et milieu de la saison des pluies (Mai ou Juin). Au total 252 relevés ont été effectués.

La nomenclature des espèces a été faite suivant celle de Lebrun et Stork (1991 - 1997).

\section{Analyse des données}

Trois types d'analyses ont été réalisés : les ordinations, les classifications et l'identification des espèces indicatrices.

L'ordination des données floristiques a été effectuée à partir d'une analyse en composantes principales (ACP) avec le logiciel MVSP (Kovach, 2005). La matrice utilisée est une matrice de similarité construite avec le calcul de l'indice de similarité de Bray et Curtis sur des données ayant subit des transformations logarithmiques.

Les données d'abondance-dominance ont été transformées en coefficient de type Van der Maarel, afin de ne pas donner trop de poids aux espèces dominantes (Dufrêne, 2004). Par ailleurs, le poids des espèces rares a été réduit à l'aide de l'option "downweighting of rare species » du logiciel MVSP (Kovach, 2005).

Vu que l'étude concerne plusieurs types de végétation, Kent et Coker (1992) ont suggéré qu'il était souvent nécessaire de fragmenter l'analyse en plusieurs états pour avoir des résultats satisfaisants. Donc, dans un premier temps, tous les relevés ont été analysés ensemble ; ce qui a permis d'avoir une vue d'ensemble de l'organisation des données. Mais après la première ordination, des partitions successives ont été effectuées jusqu'à ce que des groupes de relevés représentatifs d'un type d'habitat soient mis en évidence (Gauch, 1982).

L'étape suivante a été la classification des relevés qui a consisté à diviser les groupes mis en évidence par l'ACP. Une classification ascendante hiérarchique des relevés a été effectuée à l'aide du logiciel XL STAT 2007. La distance de Bray Curtis a été utilisée comme mesure de distance et le minimum de variance de Ward comme méthode d'agrégation.

Afin d'obtenir les espèces caractéristiques de chaque groupe obtenu avec la classification hiérarchique des relevés, la méthode d'analyse des espèces indicatrices (Indicator Species Analysis ou ISA) de Dufrêne et Legendre (1997) a été utilisée. Pour chaque groupe mis en évidence par les dendrogrammes, la valeur indicatrice ou IV de toutes les espèces s'y retrouvant a été calculée avec le logiciel IndVal 2.0 (Dufrêne, 2004). Un poids égal a été utilisé pour les deux composantes de l'index et 499 permutations ont été effectuées pour tester la valeur significative à $p<0,05$.

Par rapport aux résultats des travaux de Dufrêne et Legendre (1997), les espèces ayant une valeur indicatrice (IV) supérieure ou égale à 25 ont été retenues.

\section{RESULTATS}

\section{GRADIENTS ENVIRONNEMENTAUX ET IDENTIFICATION DES FORMATIONS VEGETALES}

L'ordination de l'ensemble des relevés à partir de la matrice de 252 relevés et de 398 espèces (Figure 2) révèle, sur l'axe 1 , un gradient de salinité qui sépare d'un côté les relevés des mangroves et de l'autre côté, vers la droite, les relevés des prairies et des forêts marécageuses d'eau douce. L'axe 2 sépare clairement les relevés en fonction du type de végétation dominant avec le noyau supérieur formé par les relevés effectués dans les zones humides à végétation herbacée dominante appelé « zones 
humides herbacées ». Le noyau en dessous regroupe les relevés effectués dans les formations à végétation ligneuse dominante appelé aussi « zones humides boisées ».

Les relevés intermédiaires ou mixtes comprenant des arbres, arbustes et quelques herbacées ont été réunis pour l'analyse des « zones humides boisées ».

L'ordination partielle des relevés des «zones humides boisées » a permis d'isoler les relevés des mangroves des relevés sur substrats non salés qui rassemblent les autres formations forestières édaphiques (Figure 3 ). L'axe 1 représente un gradient de salinité avec, à gauche, les relevés des mangroves. L'axe 2 permet de séparer les relevés suivant les types de sols, avec au-dessus, les relevés sur des sols hydromorphes organiques et en dessous, les relevés sur sols minéraux. Les relevés des forêts ripicoles qui se retrouvent dans le nuage des relevés des mangroves correspondent aux forêts ripicoles estuariennes.

L'ACP de l'ensemble des relevés des « zones humides herbacées » n'a pas permis de séparer les marais herbacés des mangroves des autres formations végétales herbacées. L'ACP des relevés des zones humides herbacées sur substrats non salés a montré une séparation nette des prairies aquatiques, des prairies marécageuses et des savanes herbeuses. Le premier axe représente un gradient d'hydropériode décroissant.

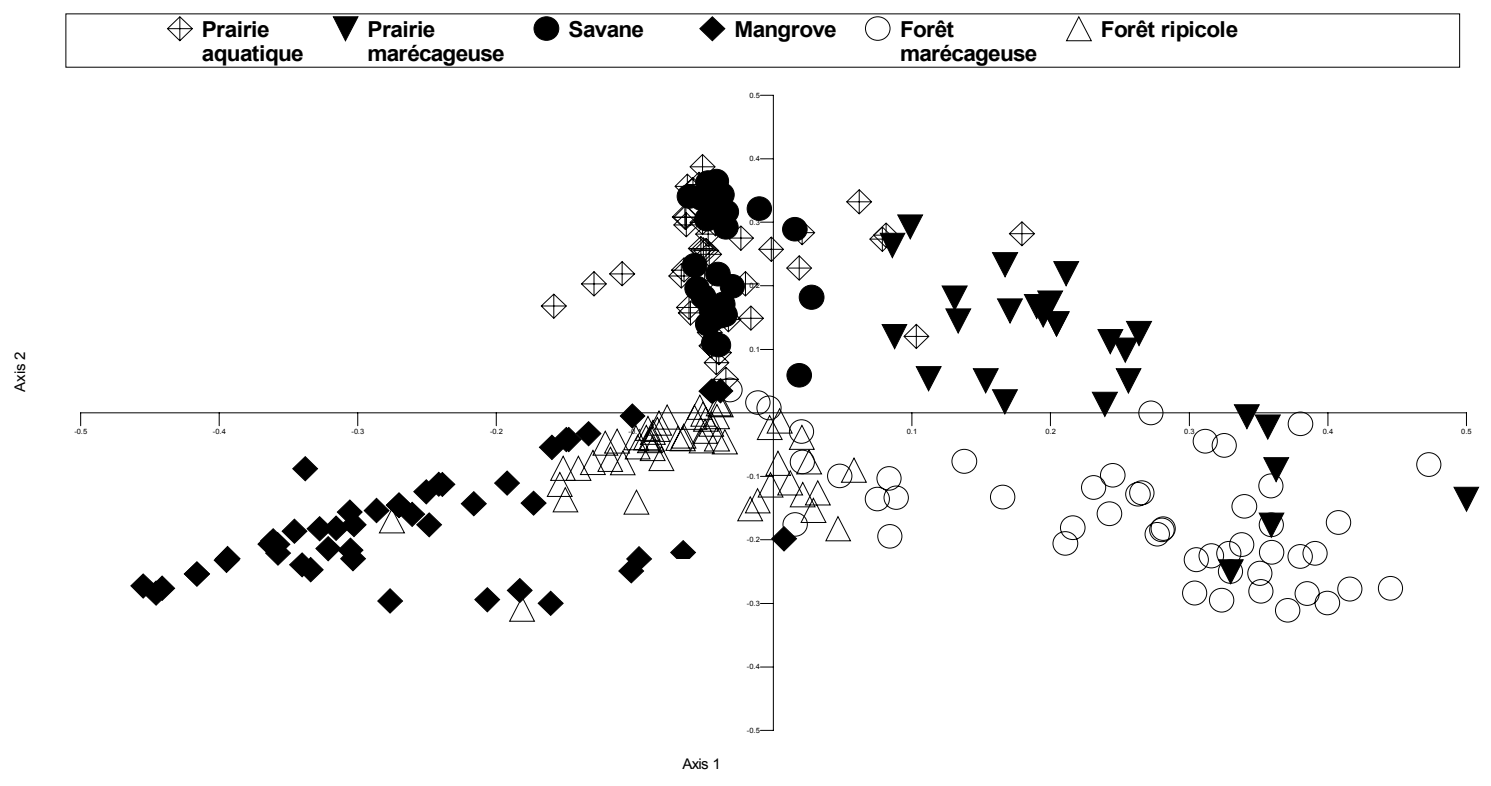

Figure 2 : Représentation des 2 premiers axes de l'ACP de l'ensemble des relevés. Les symboles utilisés sont basés sur les types de formations végétales assignés a-priori à chaque relevé

Plot of the first two axes of the floristic dataset correspondence analysis. The symbols used are based on the a-priori habitat typology 


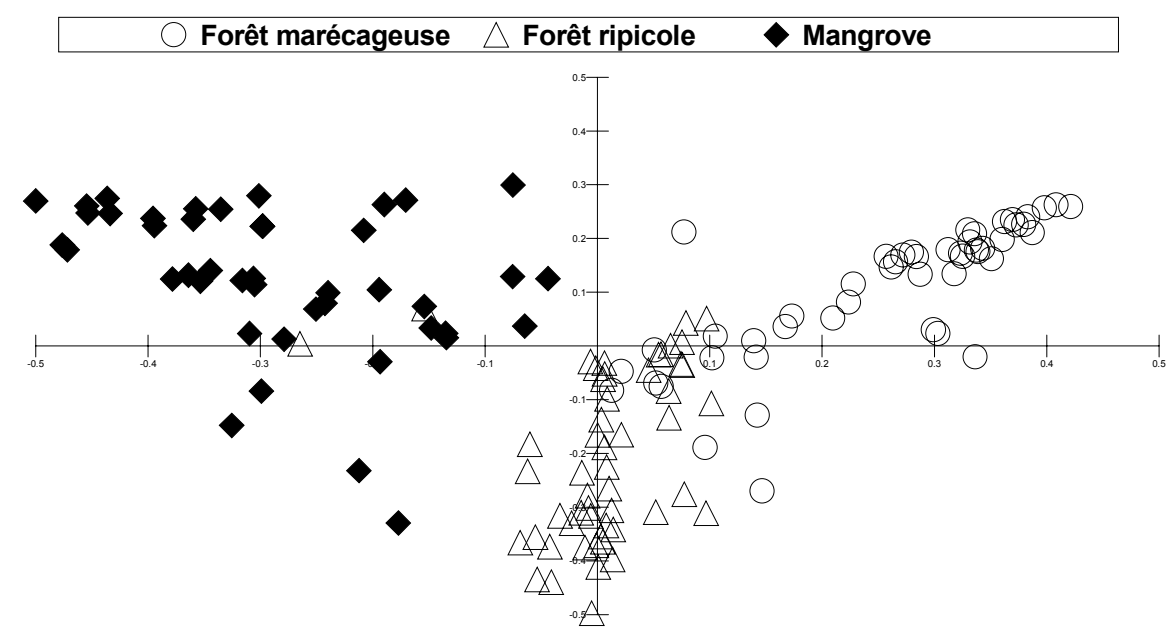

Figure 3 : Représentation des 2 premiers axes de l'ACP des 147 relevés des zones humides boisées.

Plot of the first two axes of the 147 releves of the woody wetland correspondence analysis.

\section{Classification et communautés végétales des mangroves}

Les mangroves ont été caractérisées par un dendrogramme à 5 classes a partir duquel les espèces indicatrices ont été identifiées.

Communauté végétale 1 : Rhizophora racemosa est la principale espèce indicatrice de cette communauté qui est bien développée en bordure des lagunes Aby, au niveau du Parc national des îles Ehotilés (Figure 4) et dans des stations de mangroves bien conservées au niveau de l'île Vitré en lagune Ebrié. Les sols sont limoneux, avec des sables fins sabloargileux en surface, et un $\mathrm{pH}$ acide $(4,71$ en moyenne).

Communauté végétale 2 : Les principales espèces indicatrices sont Rhizophora racemosa et Avicennia germinans. Cette formation mixte se développe sur les substrats vaseux ou sablovaseux.

Communauté végétale 3 : Elle correspond au fourré à Machaerium lunatum et Dalbergia ecastaphyllum, que l'on rencontre dans les parties dégradées des mangroves.

Communauté végétale 4 : Elle comporte les mangroves à Avicennia germinans et est souvent seule ou quelquefois associée à Acrostichum aureum et Hibiscus tiliaceus. L'influence des marées est intermittente. La texture des sols est sablo-argileuse.

Communauté végétale 5 : Elle représente une formation mixte regroupant des espèces de la zone estuarienne et des espèces communes avec les mangroves telles que Machaerium lunatum et des plantes de forêts ripicoles notamment Pterocarpus santalinoides et Crudia klainei.

\section{Classification et communautés végétales des zones humides boisées d'eau douce}

Huit communautés végétales ont été discriminées pour ce sous-groupe.

Communauté végétale 1 : Elle rassemble la flore de la végétation dégradée des bordures des lagunes. Aucune espèce n'atteint sa valeur indicatrice maximale. C'est une communauté mixte de grands arbres de forêts marécageuses ou ripicoles comme Uapaca heudelotii, Parkia bicolor, Raphia hookeri, Cathormion altissimum, Spondianthus preussii, Uapaca sp., et Ficus ovata. Cette communauté est présente en bordure de la lagune Ehy, à Allangouanou sur substrat argileux, à Kodjoboué sur substrats sableux et en bordure de la baie de N'guiémé.

Communauté végétale 2 : Cette communauté végétale se retrouve dans les sites de dépressions marécageuses qui forment un fourré dominé par Alchornea cordifolia, Macaranga heudelotii et Raphia hookeri.

Communauté végétale 3 : Elle correspond à la végétation des stations des fourrés arbustifs des berges des cours d'eau qui comportent des espèces ubiquistes et des lianes comme Cuviera macroura, Alchornea cordifolia, 
Myrianthus serratus, Tetracera alnifolia, Quisqualis indica, Paullinia pinnata, Phyllanthus reticulatus et Allophylus africanus. Les sols subissent des inondations prolongées ou brèves pendant les périodes de crues. Leur texture varie entre argileux, argilo-sableux et limoneuxargileux.

Communauté végétale 4 : Elle comporte la végétation des forêts marécageuses. Les espèces indicatrices de cette communauté sont de grands arbres comme Symphonia globulifera, Uapaca paludosa, Anthostema aubryanum, Nauclea pobeguinii et Hallea ledermannii. L'hydromorphie est permanente avec une inondation en saison des pluies sur les sols plats hydromorphes organiques. Le pH moyen est de 4,8. Ces sols ont un horizon supérieur limoneux et organique, mais la texture, en profondeur, est sablo-argileuse. Cette communauté végétale a été observée dans les dépressions marécageuses à Fatoukro et dans les bas-fonds inondables en bordure des rivières Toumanguié et Tanoé (Forêt marécageuse de la Tanoé).

Communauté végétale 5 : Cette communauté végétale se met en place sur les substrats marécageux dominés par Hallea ledermannii, Raphia hookeri et Spondianthus preussii. On les rencontre dans les marécages à N'gandaN'ganda et les forêts marécageuses à Fatoukro.

Communauté végétale 6 : Raphia hookeriest la principale espèce avec un recouvrement compris entre $75-100 \%$ dans certains relevés, formant ainsi des raphiales. Cette communauté se rencontre dans les stations de forêts marécageuses fortement dégradés à NgandaNganda et notamment dans les marécages de la Tanoé.

Communauté végétale 7 : Cette communauté végétale correspond aux prairies marécageuses dominées par les arbustes tels que Ficus trichopoda, Voacanga thouarsii, Raphia hookeri et Stipularia africana. La strate herbacée comprend principalement Lasiomorpha senegalense et Cyclosorus striatus. Cette communauté se rencontre en lagune Kodjoboué, en baie de N'guiémé et dans les dépressions marécageuses sur substrat hydromorphe organique inondé en permanence des sites de forêts marécageuses dégradés.

Communauté végétale 8 : Elle correspond aux formations ligneuses des berges des cours d'eau. On la rencontre notamment en bordure de la Comoé (pentes abruptes à Moossou et relativement douces à Motobé), de la Bia et de la Tanoé (pentes abruptes et substrat argileux). Le substrat est argileux en bordure de la lagune Ono à Adiaho. A Motobé, le pH moyen est de 4,8, à Eholié il est de 4,31 et à Ehania de 5,23. Les espèces indicatrices comprennent Pterocarpus santalinoides, Uapaca heudelotii, Cathormion altissimum, Crudia klainei et Treculia africana. Ces espèces sont observées en premier plan au bord de l'eau (sur les berges des fleuves).

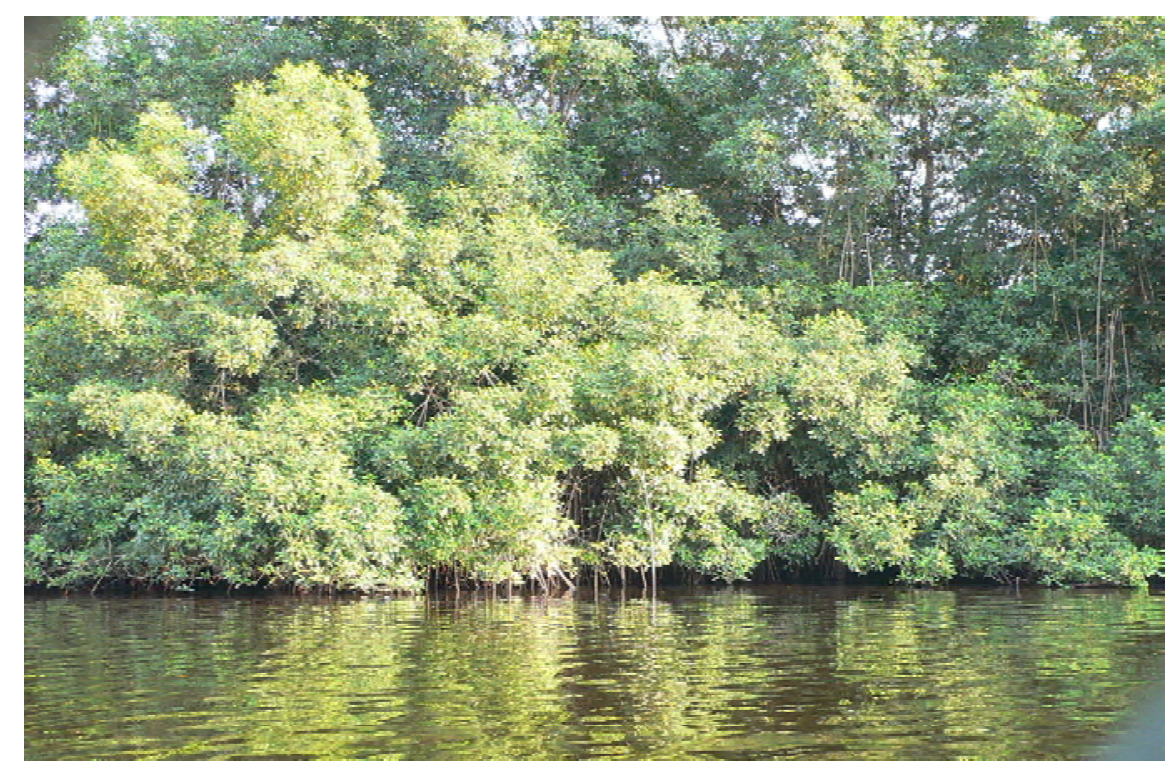

Figure 4 : Rideau de Rhizophora racemosa au Parc National des lles Ehotilés (Egnankou Wadja Mathieu, 2007).

Rhizophora racemosa at Ehotilés Islands National Parc. 


\section{Classification et communautés végétales des zones humides herbacées}

L'analyse des espèces indicatrices montre que le dendrogramme à 14 classes de la classification ascendante hiérarchique des flores des formations herbacées donne le maximum de valeurs indicatrices significatives à $P<0,05$ (196). Cependant, dans l'interprétation des attributs de ces classes, il semble nécessaire de regrouper certaines classes, surtout quand celles-ci ne représentent qu'une variation floristique sans caractéristique environnementale particulière. Finalement, 9 types de communautés végétales ont été retenus pour caractériser des zones humides herbacées du Sud-Est de la Côte d'Ivoire.

Communauté végétale 1: Elle compose la végétation des marais d'eau douce. Cette communauté a été reconnue dans les mares temporaires des savanes herbeuses sur substrat sableux à Moossou. Le pH varie entre 5 et 6 et les eaux sont douces. L'espèce distinctive de cette communauté est Aniseia martinicensis.

Communauté végétale 2: Elle compose la végétation des savanes herbacées littorales. On la rencontre à Moossou et à N'ganda-N'ganda sur les sols blanchâtres à texture sableuse (pseudo-podzol de nappe) et à un $\mathrm{pH}$ moyen de 4,86 . La nappe phréatique étant proche de la surface du sol, ces savanes connaissent de brèves inondations durant la saison des pluies. Ces savanes présentent un faciès dominé par Loudetia phragmitoides et Anadelphia erecta dont le recouvrement atteint $95 \%$. On y rencontre aussi des bosquets dominés par Clappertonia ficifolia, et des fourrés marécageux avec Stipularia africana, Ficus trichopoda et Cyclosorus striatus.

Communauté végétale 3 : Cette communauté végétale correspond aux marais saumâtres bas. Elle se rencontre en arrière des mangroves ou dans ses zones dégradées, sur des sols argileux, sableux compacts et salés. L'hygrométrie varie selon la saison : submergées en période pluvieuse et fortement desséchées durant la saison sèche. Les espèces indicatrices sont : Paspalum vaginatum, Cyperus articulatus, Mariscus ligularis, Sesuvium portulacastrum et Blutaparon vermicularis. Paspalum vaginatum forment des pelouses monospécifiques. Quelques jeunes plants de Rhizophora racemosa s'observent dans cette communauté végétale. Cette communauté a été reconnue à l'estuaire de Grand Bassam, dans les alentours de l'île Vitrée, à Ebrah, et au niveau du Parc National des îles Ehotilé notamment sur l'île Balouhaté.

Communauté végétale 4 : Elle comprend les mares temporaires ou stagnantes dominées par les hydrophytes enracinées à feuilles étalées sur l'eau que sont Nymphaea lotus et Nymphaea maculata. Ces mares s'enrichissent en hélophytes telles que Ludwigia stolonifera et Eleocharis mutata. Cette communauté a été observée dans les mares temporaires des savanes herbeuses sur substrat sableux à Moossou et les mares ou petites dépressions permanentes isolées ou les bordures calmes des plans d'eau. Le substrat de fonds est généralement sableux et le $\mathrm{pH}$ varie entre 5 et 6 . Les eaux sont douces.

Communauté végétale 5 : Elle est dominée par Lemna aequinoctialis. On la rencontre sur les petites retenues isolées peu profondes souvent associés à Wolffia arrhiza et à Azolla africana.

Communauté végétale 6 : Cette communauté végétale est dominée par Typha domingensis qui forme des roselières dans les zones estuariennes, notamment à Grand Bassam dans la lagune Ouladine, à la sortie du Pont de la Victoire. Les sols sont argileux, toujours inondés et les eaux sont salées à moyennement salées, en fonction de la saison et de l'état de l'embouchure. En période d'ouverture de l'embouchure, en novembre 2004, une salinité de $25 \%$ a été mesurée. Les autres espèces associées à ces roselières saumâtres sont : Cyperus articulatus, Vossia cuspidata, Echinochloa pyramidalis.

Communauté végétale 7 : Elle correspond à la végétation flottante, avec comme noyau floristique de base, les macrophytes envahissants comme Eichhornia crassipes, Salvinia molesta et Pistia stratiotes. C'est une communauté végétale quelquefois monospécifique. Les espèces fréquemment associées sont Bacopa crenata et Ipomoea aquatica. Cette communauté se rencontre sur toutes les lagunes et les fleuves de la zone d'étude.

Communauté végétale 8 : Elle correspond aux prairies marécageuses des berges lagunaires ou des dépressions marécageuses. Les espèces de base de cette communauté sont 
les hélophytes tels que Echinochloa pyramidalis ou Vossia cuspidata et les géophytes rhizomateux tels que Cyclosorus striatus et Lasiomorpha senegalense. Des espèces ligneuses comme Ficus trichopoda, Voacanga thouarsii et Stipularia africana apparaissent plus tard sur les substrats plus épais. On rencontre ces prairies flottantes sur le plan d'eau des lagunes Tendo à N'guiémé, Ebouinda et Kodjoboué. Le substrat est hydromorphe organique et en bordure des lagunes, il est composé de débris végétaux. Le $\mathrm{pH}$ varie entre 4,97 et 5,4 .
Communauté végétale 9 : L'espèce caractéristique de ce groupe est Echinochloa pyramidalis qui forme des roselières fluviatiles et lagunaires souvent monospécifiques. On y rencontre d'autres espèces telles que Eichhornia crassipes, Leersia hexandra et Pistia stratiotes. Ces roselières sont observables en bordure de presque tous les cours d'eau de la région du Sud-Comoé, notamment le long du fleuve Comoé à Grand-Bassam (Figure 5), de la lagune Ono et en lagune Aby du secteur Ehy à Tendo.

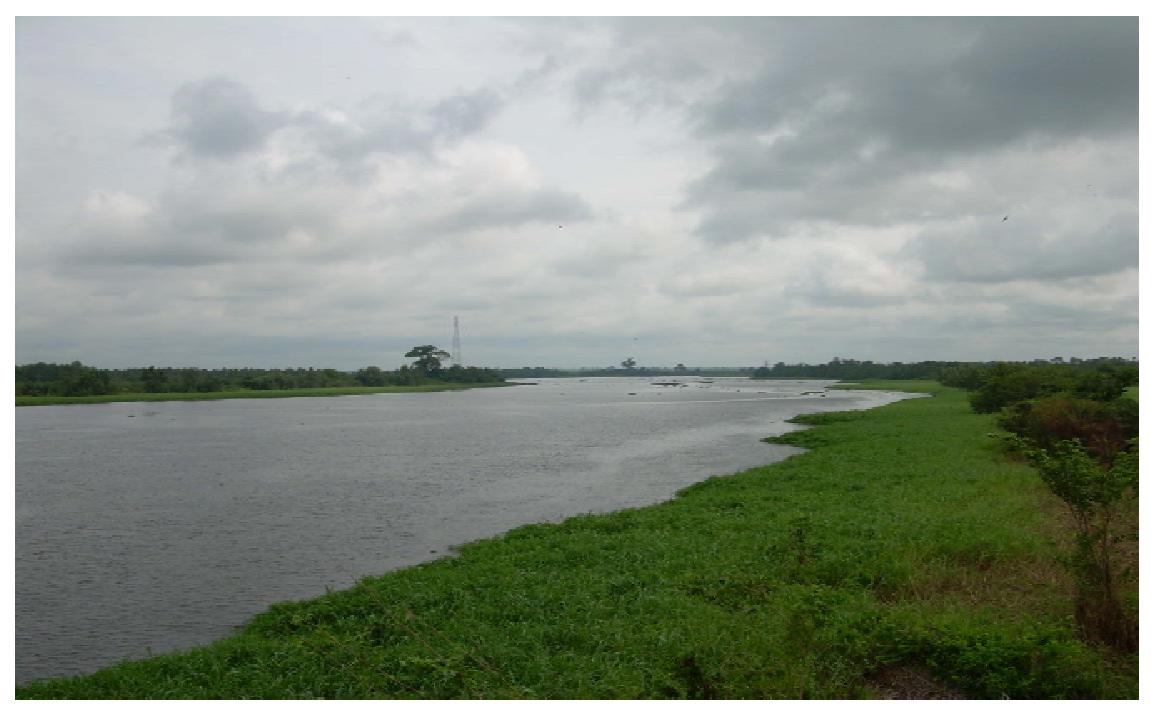

Figure 5 : Roselières à Echinochloa pyramidalis le long du fleuve Comoé à Grand-Bassam (Kouame Ode Marie Louise, 2008)

Prairies of Echinochloa pyramidalis along the Comoe River in Grand-Bassam

\section{DISCUSSION}

Les analyses successives ont abouti à la séparation des formations végétales et à l'identification des gradients environnementaux de la végétation.

La stratification à-priori des relevés dans les catégories de végétation de la classification de Yangambi (Aubréville, 1957) a été confirmée par l'ordination.

Orthmann (2005) dans son étude sur les mosaïques savane-forêt du centre du Bénin a procédé par une stratification suivant les types physionomiques de Yangambi (Aubréville, 1957) et par une classification phytosociologique. Ses résultats ont montré une meilleure discrimination au sein des groupes phytosociolologiques que des groupes physionomiques.

Dans la présente étude, les ordinations ont seulement donné un aperçu général de la séparation des relevés et ont confirmé le regroupement selon les catégories physionomiques de végétation de Yangambi (Aubréville, 1957). Les ordinations uniquement n'auraient pas permis de relier les caractéristiques physionomiques à la composition en espèces des communautés végétales. Ce qui a été rendu possible grâce aux méthodes de classification et d'analyse des espèces indicatrices. 
Menaut (1983) a suggéré que pour une comparaison et une compréhension globale de la végétation, les catégories de Yangambi (Aubréville, 1957) soient utilisées. Mais il ajoute qu'il faudrait par la suite une classification phytosociologique plus détaillée à l'intérieur de chaque catégorie de Yangambi.

Ceci a été appliqué par Poilecot et al. (1991) au nord-est de la Côte d'Ivoire et par Orthman (2005).

Hupalo (2000) a stratifié les zones humides du Colorado suivant les sous-classes hydro géomorphologiques de Brinson et al. (1995). Brinson et al. (1995) a mis en évidence que les facteurs hydrologiques et géomorphologiques influencent le fonctionnement des zones humides. II reconnait aussi que les communautés de plantes des zones humides sont aussi indicatrices des forces hydrogéomorphiques affectant ces écosystèmes.

Hupalo (2000) a aboutit a l'identification d'espèces indicatrices pour les différentes classes hydrogéomorphologiques de Brinson et al. (1995) que sont zones humides de dépression, fluviales, de pente et d'étendue plate.

L'approche de Hupalo (2000) pourrait être utilisée avec efficacité avec des données plus importantes et à plus grande échelle pour l'étude des zones humides de la Côte d'Ivoire.

L'ordination et la classification ont montré que la végétation des zones humides est structurée le long de gradients que sont le type de végétation dominante, la salinité, le type de sol et l'hydromorphie.

L'interprétation des gradients environnementaux responsables de la typologie des communautés végétales des zones humides est proche des observations faites par d'autres travaux portant sur la végétation hydrophytique.

En effet, Nygaard (2004) considère le régime hydrologique comme l'un des plus importants facteurs dans les zones humides.

Pour Lebrun et al. (1954), à côté de la variation du régime d'inondation, l'intensité du drainage, la physiographie des sites, le degré de l'atterrissement ou de l'alluvionnement, la nature de l'eau et du substrat jouent aussi un rôle dans la typologie de la végétation hydrophytique.

De façon plus particulière, pour les forêts ripicoles, il a été montré par Sokpon et al. (2001) que la durée de la période de ressuyement ou de l'inondation, la latitude et la topographie sont les facteurs qui discriminent aux mieux les forêts édaphiques du Bénin. Natta (2002) a aussi considéré que les 5 facteurs responsables de la variabilité des forêts ripicoles au Bénin sont le relief, le type et l'importance des cours d'eau, la topographie, la latitude et la longitude.

Les méthodes d'analyses multivariées utilisées permettent de confirmer les facteurs responsables de la discrimination des formations végétales mises en évidence par les méthodes phytosociologiques classiques. En effet, d'une manière générale, les résultats des $A C P$, des dendrogrammes et de l'analyse des espèces indicatrices (ISA) sont complémentaires et efficaces pour la partition des groupes de relevés basés sur leur composition floristique.

Vingt deux communautés végétales ont été décrites dans la présente étude. Les communautés végétales des zones humides herbacées comprennent des espèces indicatrices qui peuvent se ranger dans la Classe des Phragmitetea Tüxen \& Pressing 1942 et la Classe de Potametea Tuxen et Preising 1942. Des espèces appartenant aux subdivisions «Alliance» de ces Classes ont ainsi été reconnues :

- espèces indicatrices de l'Alliance de l'Echinochloion tropicale avec Echinochloa pyramidalis ;

- espèces indicatrices appartenant à l'Alliance du Magnocyperion africanum Lebrun 1947 comme Polygonum salicifolium, Rhynchospora corymbosa et Cyperus articulatus ;

- espèces indicatrices caractéristiques de I'Alliance du Papyrion Lebrun 1947 et de I'Alliance du Jussieuon Léonard 1950 comme Cyclosorus striatus et Lasiomorpha senegalense.

En ce qui concerne les communautés végétales des zones humides boisées, elles peuvent se ranger dans la Classe des Mitragynetea de Schmitz (1963). Les espèces indicatrices appartiennent aux subdivisions suivantes:

- espèces indicatrices de l'Alliance Alchorneion cordate comme Ficus trichopoda, Stipularia africana et des espèces indicatrices de l'Alliance Uapacion heudelotii Lebrun et Gilbert 1954 ;

- espèces indicatrices de l'Alliance Raphion Devred 1958 et de l'Alliance MitragynoSymphonion Devred 1958. 


\section{CONCLUSION}

Les analyses numériques effectuées avec les 252 relevés et 398 espèces ont révélés que les communautés végétales des zones humides du Sud-Est de la Cote d'Ivoire se discriminent suivant le type de végétation dominante, la salinité, le type de sol et l'hydromorphie.

Cette étude donne un aperçu des gradients de végétation dans les zones humides naturelles peu perturbées.

D'autres études, un peu plus importantes et approfondies dans le cadre d'un inventaire national des zones humides, sont nécessaires pour une évaluation et une classification complètes et prédictibles des types de végétation des zones humides de la Côte d'Ivoire. Elles devront intégrer des modificateurs tels que les changements induits par des facteurs anthropiques ou les phénomènes environnementaux naturels comme les inondations, les modifications hydrologiques et les projets de développement de la région tels que la construction du Centre de Biotechnologie à Grand-Bassam.

\section{REFERENCES}

Adjanohoun E. 1965. Comparaison entre les savanes côtières de la Côte d'Ivoire et du Dahomey. Ann. Univ. Abidjan, série Sciences (1) : $41-62$.

Adomou A. C. 2005. Vegetation patterns and environmental gradients in Benin : implications for biogeography and conservation. PhD thesis, Wageningen University, Wageningen, $150 \mathrm{p}$.

Afidegnon D. 1999. Les mangroves et les formations associées du Sud-Est du Togo : Analyse éco-floristique et cartographie par télédétection spatiale. Thèse de doctorat, Université du Bénin, spécialité Botanique, $212 \mathrm{p}$.

Allen R. B. and R. K. Peet. 1990. Gradient analysis of forests of the Sangre de Cristo Range, Colorado. Canadian Journal of Botany 68 : $193-201$.

Aubréville A. 1957. Accord à Yangambi pour la nomenclature des types africains de végétation. BFT $51: 22$ - 27.
Brinson M. M., Hauer F. R., Lee L. C., Nutter W. L., Rheinhardt R. D., Smith R. D. and Whigham D. F. 1995. A guidebook for application of hydrogeomorphic assessments to riverine wetlands, USACE, Waterways Experiment Station, Technical Report WRP - DE - 11, $113 \mathrm{p}$

Dufrêne M. 2004. The IndVal Program version 2.0 http://mrw.wallonie.be/dgrne/sibw/outils/ home.html.

Dufrêne M. and L. Legendre. 1997. Species assemblages and indicator species : the need for a flexible asymmetrical approach. Ecological Monographs $67: 345$ - 366.

Ecological Society of America. 2004. Guidelines for describing associations and alliances of the US National Vegetation Classification, version 4.0, July 2004, 165 p.

Egnankou W. M. 1985. Etude des mangroves de Côte d'Ivoire : aspect écologique et recherches sur les possibilités d'aménagement. Thèse de Doctorat $3^{\text {ème }}$ cycle, Univ. Paul Sabatier de Toulouse, Série Sciences (3196), $167 \mathrm{p}$.

Gauch H. G. 1982. Multivariate analysis in community ecology. Cambridge University Press, London, UK, 298 p.

Guillaumet J. L. 1967. Recherches sur la végétation et de la flore de la région du Bas-Cavally (Côte d'Ivoire). Mém. ORSTOM 20 : 249 p.

Hoagland B. W. 2002. A Classification and Analysis of Emergent Wetland Vegetation in Western Oklahoma Proc. Okla. Acad. Sci. 82 : 5 - 14.

Houenon J. 1989. Ecologie, végétation et flore des rives du système lagunaire Ebrié. Mém. DEA, Faculté de Sciences, Université Nationale de Côte d'Ivoire, 155 p.

Hupalo J. 2000. Data Compilation and Stratification for a Wetland Plant Association Classification of Colorado. Colorado Natural Heritage Program, College of Natural Resources, Colorado State University, $49 \mathrm{p}$.

Kent M. and P. Coker. 1992. Vegetation description and analysis : a practical approach. Belhaven Press. London, UK, 363 p.

Kimmins J. P. 1997. Forest ecology : a foundation for sustainable management. Second edition. Prentice Hall, Upper Saddle River, New Jersey, USA, 596 p.

Kokou K. 1998. Les mosaïques forestières au Sud du Togo: biodiversité, dynamique et activités humaines. Thèse Doct. d'Etat, Univ. Montpellier II (France), 140 p. 
Konan-Brou A. A., Traoré D., Sankaré Y. et N. Ettien. 1992. Etude de la ceinture hydrophytique du secteur II de la lagune Ebrié (Côte d'Ivoire). Agron. Afri. 4 : 87 - 178.

Kouamé O. M. L. 1999. Flore, végétation et Ecologie de la baie de N'guiémé en lagune Tendo (Sud-Est de la Côte d'Ivoire. Mém. DEA, Université d'Abidjan-Cocody, $174 \mathrm{p}$.

Kovach W. L. 2005. MVSP - A MultiVariate Statistical Package for Windows, ver. 3.1. Kovach Computing Services, Pentraeth, Wales, U. K.

Lebrun J. P. et A. Stork. 1991. Enumération des plantes à fleurs d'Afrique tropicale. I - Généralités et Annonaceae à Pandaceae, Conservatoire et Jardin botaniques de la ville, Genève, $250 \mathrm{p}$.

Lebrun J. P. et A. Stork. 1992. Enumération des plantes à fleurs d'Afrique tropicale. II - Chrysobalanaceae à Apiaceae, Conservatoire et Jardin botaniques de la ville, Genève, 260 p.

Lebrun J. P. et A. Stork. 1995. Enumération des plantes à fleurs d'Afrique tropicale. III - Monocotylédones : Limnocharitaceae à Poaceae, Conservatoire et Jardin botaniques de la ville, Genève, $340 p$.

Lebrun J. P. et A. Stork. 1997. Enumération des plantes à fleurs d'Afrique tropicale. IV - Gamopétales : Clethraceae à Lamiaceae, Conservatoire et Jardin botaniques de la ville, Genève, $712 \mathrm{p}$.

Mack J. J. 2004. Integrated Wetland Assessment Program. Part 2 : an ordination and classification of wetlands in the Till and Lake Plains and Allegheny Plateau regions. Ohio EPA Technical Report, WET/2004-2. Ohio Environmental Protection Agency, Wetland Ecology Group, Division of Surface Water, Columbus, Ohio, 76 p.

McCune B., J. B. Grace and D. L. Urban. 2002. Analysis of ecological communities. MjM Software Design, Gleneden Beach, Oregon, USA, $300 \mathrm{p}$.

Menaut J.-C. 1983. The vegetation of African savannas. In : Bourliere, F. (ed.) Tropical Savannas, Elsevier, Amsterdam : pp $109-149$

Mueller-Dombois D. and H. Ellenberg. 1974. Aims and Methods of Vegetation Ecology. John Wiley \& Sons, New York, 547 p.
Natta A. K. 2003. Ecological assessment of riparian forests in Benin. Phytodiversity, phytosociology and spatial distribution of tree species. PhD thesis, Wageningen University, The Netherlands, $215 \mathrm{p}$.

Nygaard B. 2004. Community assembly in restored wetlands. PhD thesis, National Environ mental Research Institute, Kalo, Denmark, $40 \mathrm{p}$.

Orthmann B. 2005. Vegetation ecology of a woodland-savanna mosaic in central Benin (West Africa) : Ecosystem analysis with a focus on the impact of selective logging Rostock, Univ., Diss., 148p.

Podani J. 2000. Introduction to the exploration of multivariate biological data. Backhuys Publishers, Leiden, Hungary, 407 p.

Poilecot P., Bonfou K., Dosso H., Lauginie F., N'Dri K., Nicole M. et Y. Sangaré. 1991. Un écosystème de savane soudanienne : le Parc National de la Comoé (Côte d'Ivoire). UNESCO, Paris ; 346 p.

Sankaré Y., Avit J.-B. L. F., Egnankou W. et P. Saenger. 1999. Etude floristique des mangroves des milieux margino-littoraux de Côte d'Ivoire. Bull. Jard. Bot. Belg. 67 : $335-360$.

SECA-BRL/BDPA/RCT. 2004. Livre Blanc du Littoral ivoirien. Ministère des Affaires Etrangères Français, $46 \mathrm{p}$.

Senterre B. 2005. Recherches méthodologiques pour la typologie de la végétation et la phytogéographie des forêts denses d'Afrique tropicale. Thèse de Doctorat, Sciences Agronomiques et Ingénierie biologique, Université Libre de Bruxelles (ULB), $345 \mathrm{p}$.

Shimwell D. W. 1971. Description and classification of vegetation. Sidgwick and Jackson, London, 322 p.

Sinsin B. 1993. Phytosociologie, écologie, valeur pastorale, production et capacité de charge des pâturages du périmètre Nikki-Kalalé au Nord-Bénin. Thèse de doctorat, Fac. Sc., Lab. Bot., Syst. \& Phytosoc., Université Libre de Bruxelles (ULB), $390 \mathrm{p}$.

Sokpon N., Sinadouwirou Th., Gbaguidi F. et S. H. Biaou. 2001. Aperçu sur les forêts édaphiques hygrophiles du Bénin. Belg. J. Bot. 134 (1) : 64 - 78. 
Sopkon N. 1995. Recherches écologiques sur la forêt dense semi-décidue de Pobè au SudEst du Bénin : groupements végétaux, structure, régénération naturelle et chute de litière. Thèse Doct., Univ. Bruxelles (Belgique), $350 \mathrm{p}$.

Traoré D. 1985. Contribution à l'étude des milieux hydrophytiques ouverts de la Côte d'Ivoire. Thèse de Doctorat d'Etat ès Sciences Naturelles, Univ. Bordeaux III, 394 p.
Van der Maarel E., I. Espejel and P. MorenoCasasola. 1987. Two-step vegetation analysis based on very large data sets. Vegetation $68: 139$ - 43 .

Whittaker R. H. and H. G. Gauch. 1973. Evaluation of ordination techniques. In : R. H. Whittaker (Ed.). Ordination and Classification of Communities. Junk, The Hague, Handbook of Vegetation Science 5 : 287 - 321. 